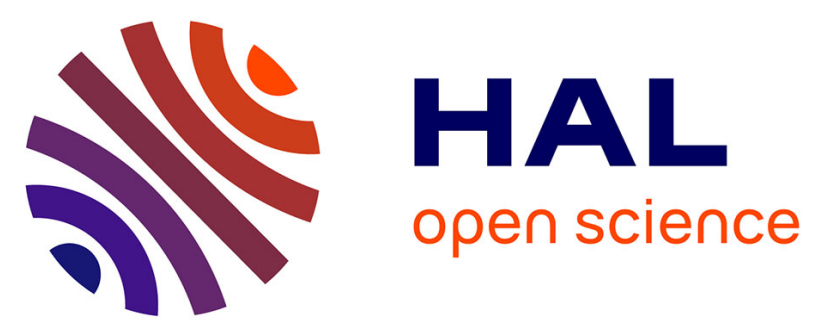

\title{
Improving EIT-based visualizations of two-phase flows using an Eigenvalue correlation method
}

Chunhui Dang, Mathieu Darnajou, Cedric Bellis, Guillaume Ricciardi, Saba Mylvaganam, Salah Bourennane

\section{To cite this version:}

Chunhui Dang, Mathieu Darnajou, Cedric Bellis, Guillaume Ricciardi, Saba Mylvaganam, et al.. Improving EIT-based visualizations of two-phase flows using an Eigenvalue correlation method. IEEE Transactions on Instrumentation and Measurement, 2021, 70, pp.1-9. 10.1109/TIM.2021.3056736 . hal-03370804

\section{HAL Id: hal-03370804 \\ https://hal.science/hal-03370804}

Submitted on 8 Nov 2021

HAL is a multi-disciplinary open access archive for the deposit and dissemination of scientific research documents, whether they are published or not. The documents may come from teaching and research institutions in France or abroad, or from public or private research centers.
L'archive ouverte pluridisciplinaire HAL, est destinée au dépôt et à la diffusion de documents scientifiques de niveau recherche, publiés ou non, émanant des établissements d'enseignement et de recherche français ou étrangers, des laboratoires publics ou privés. 


\title{
Improving EIT-Based Visualizations of Two-Phase Flows Using an Eigenvalue Correlation Method
}

\author{
Chunhui Dang $^{(}$, Mathieu Darnajou ${ }^{\circledR}$, Cédric Bellis ${ }^{\circledR}$, Guillaume Ricciardi ${ }^{\circledR}$, \\ Saba Mylvaganam ${ }^{\circledR}$, Life Senior Member, IEEE, and Salah Bourennane ${ }^{\circledR}$, Member, IEEE
}

\begin{abstract}
Gas-liquid two-phase flows are encountered in various industrial processes involving high temperatures and high pressures, which necessitates nonintrusive sensing for real-time imaging of phase distribution and flow parameters. In this context, this article presents an electrical impedance tomography (EIT)-based eigenvalue correlation method that allows extracting two-phase flow features, namely, the void fraction and the flow regime, which are used in turn to improve flow visualizations. Benefiting from the so-called full-scan excitation strategy, the eigenvalue correlation method has been devised in to estimate the phase fraction from EIT raw measurements. In this article, this method is refined and integrated into an image-enhancing procedure, which is illustrated and validated using dynamic experimental data. A total of 80 experiments are considered with water and air mass flow rates ranging from 1.58 to $79.43 \mathrm{~kg} / \mathrm{min}$ and from 0.1 to $5.0 \mathrm{~kg} / \mathrm{min}$, respectively, covering slug, plug, stratified smooth, stratified wavy, and annular flows. Based on a preliminary system calibration and a raw image guess, the volume-averaged void fractions are then estimated using the proposed method and integrated into EIT-based images to form binarized tomograms relative to the acquisition time. The EIT tomograms, thus, obtained show an excellent agreement with some $\gamma$-ray reference measurements of the phase distribution.
\end{abstract}

Index Terms-Eigenvalues, electrical impedance tomography (EIT), impedance matrix, tomogram, void fraction estimation.

\section{INTRODUCTION}

G AS-LIQUID two-phase flows play a vital role in various industrial processes, for example, in the electricity generation industry where the flow transfers heat from a power core to a generating turbine. In such processes, it is crucial to optimize performances by detecting flow regimes and gas build-ups as potential escalations can compromise the safety of operations. In the nuclear power industry, in particular,

Chunhui Dang, Mathieu Darnajou, and Guillaume Ricciardi are with CEA-DES-IRESNE-DTN-LTHC, CEA Cadarache, 13115 Saint-Paul-lèsDurance, France (e-mail: dang.chunhui@ centrale-marseille.fr).

Cédric Bellis is with CNRS, Centrale Marseille, LMA, Aix-Marseille University, 13007 Marseille, France.

Saba Mylvaganam is with the University of South-Eastern Norway, 3679 Notodden, Norway.

Salah Bourennane is with the École Centrale de Marseille, 13013 Marseille, France. evaluations of heat transfer and multiphase flow instabilities in reactors are critical to safety [1]. In this context, there is a great need for nonintrusive instrumentation techniques for online monitoring of the gas-liquid two-phase flows.

Up until now, a number of tomographic techniques have been developed and assessed in terms of the accuracy of the void fraction estimation and the complexity of their practical implementation. For example, X-ray or $\gamma$-ray tomography allow fast flow measurements at the high spatial resolution, but they require high acceleration voltage (hundreds of $\mathrm{kV}$ ) and radiation protection [2]; wire-mesh sensors can provide information about local, cross-sectional or in situ volume profiles, and phase distributions, but they have disruptive effects on the flow [3]; and electrical impedance/capacitance tomography (EIT/ECT) determines the conductivity/permittivity distribution inside a domain from measurements at its boundary, leading to reconstruction profiles with relatively low spatial resolution, which can, however, be counterbalanced by high-speed implementations. Moreover, EIT attracts special attention due to its low excitation amplitudes (of the order of $\mathrm{mA}$ or few $\mathrm{V}$ ), making it safe and low cost. With these advantages, the EIT technique has been successfully applied to multiphase flow instrumentation [4], [5] and medical imaging [6].

A number of EIT systems dedicated to multiphase flow measurements have been developed. A comprehensive review of EIT applications to various configurations in the field of chemical engineering can be found in [7]. More specifically, for two-phase flow measurements, George et al. [8] presented an EIT system applied to solid-liquid and gas-liquid flows and the phase fractions within a circular cross section being determined and compared with nominal values. Jia et al. [9] developed an EIT-based measurement system for highly conductive water-oil two-phase flows, leading to cross-sectional tomographic images and tomograms, with computation of the total phase fractions and comparison to reference values. Dupré [10] developed an efficient EIT system for void fraction estimation of two-phase flows, in which the so-called full-scan excitation strategy is implemented, but only static tests were carried out to validate it.

Given the diversity of the EIT systems used in two-phase flow measurement, the full-scan excitation strategy has some distinctive capabilities. It is more advantageous compared to the adjacent strategy, which is applied in most of the practical EIT systems, in terms of robustness to measurement noise 
and quality of the reconstructed images [11]. Moreover, in the full-scan strategy, all the possible independent pairs of electrodes are used for the excitation, yielding a lower acquisition frame rate but more redundant information compared to the adjacent strategy. Taking advantage of this redundancy in the measurements, some eigenvalue-based approaches have been developed (see [12] and [13]) to estimate the phase fraction directly from a principal component analysis of raw EIT data, i.e., circumventing the image reconstruction step. This article builds from the novel eigenvalue correlation method introduced in [13], portraying new methods of sensor data fusion and image-enhancement techniques not found in the existing literature. Specifically, it describes the eigenvalue-based flow regime identification method and provides a soundproof concept related to its applicability using a pilot-scale multiphase flow rig enabling data acquisition from extensive experiments with different flow regimes. The advantages of the full-scan strategy are exploited to extract flow-related features, namely, the void fraction and the flow regime, which are used, in turn, to improve the visualizations of air-water two-phase flows.

To the best of our knowledge, there is no report of an EIT system employing the full-scan excitation strategy for dynamic two-phase flow measurements. Recently, an EIT system implementing this strategy has been developed in the Laboratory of analytical Thermo-hydraulics and Hydro-mechanics of Core and Circuits (LTHC), CEA, France, and mounted in the horizontal air-water two-phase flow loop of the University of South-Eastern Norway (USN) to perform dynamic experiments at various flow regimes. In this context, the objective of this article is to apply the proposed novel eigenvalue correlation method for dynamic two-phase flow feature extraction and to demonstrate its integration into a flow visualization improvement procedure.

The flow rig at USN, the test matrix, and the experiment are described in Section II. The image reconstruction algorithm and the proposed eigenvalue correlation method are introduced in Section III. Then, Section IV focuses on the flow visualization enhancing procedure: the eigenvalue correlation method is applied: 1) to obtain a calibration diagram relating the leading eigenvalue of the mean normalized impedance matrix to the reference volume averaged void fraction (AVF) and 2) identify the flow regime based on a raw image guess. EIT tomograms are finally obtained by axially stacking the cross-sectional image reconstructions, in which an estimated AVF is used as a threshold to improve the visualization of the phase distribution.

\section{EXPERIMENTAL StUdy CASE}

\section{A. Multiphase Flow Rig}

Air-water two-phase flow experiments were carried out in the multiphase flow rig of USN. The flow rig, as shown Fig. 1, enables the injection of oil, water, and air in a horizontal or inclined pipe at room temperature. The injection of each phase is controlled and monitored independently. The pipe has an inner diameter of $51 \mathrm{~mm}$, a thickness of $2.5 \mathrm{~mm}$, and a length of $15 \mathrm{~m}$. The mediums are mixed at the inlet of the pipe so that the flow is well developed at the observation and measurement sections. A transparent polymethylmethacry-

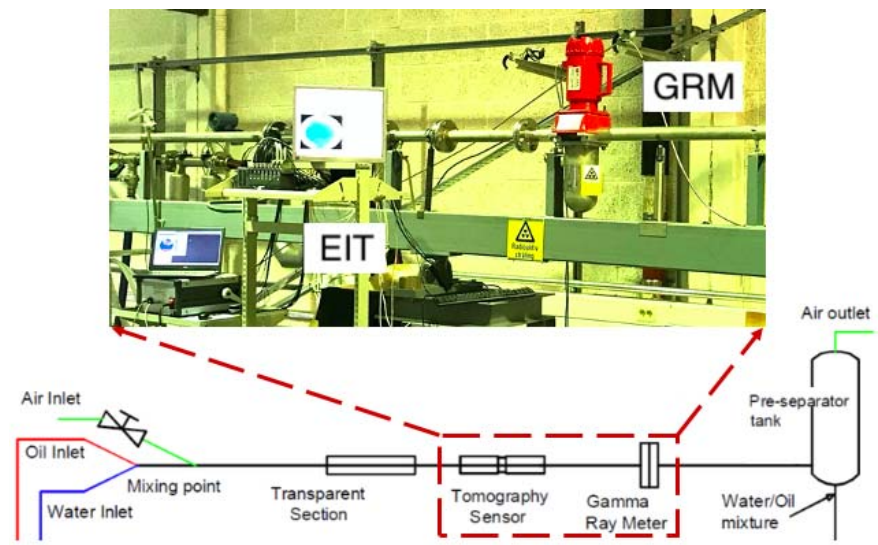

Fig. 1. Horizontal multiphase flow rig at USN.

late (PMMA) section permits visual or (high-speed) video observations to appreciate the flow regime. In addition, the rig is equipped with EIT and gamma-ray meter (GRM) devices downstream of the PMMA section.

The schematic of the EIT full-scan excitation strategy is shown in Fig. 2(a): all electrodes are sequentially selected to be the source and drain until all possible pairs of electrodes have been considered. Typically, a multiplexer is used to route the excitation signals to each independent pair of electrodes, and measurements are taken simultaneously at all electrodes. This strategy includes the excitation patterns of the adjacent and opposite strategies, among others, thus leading to comparatively longer acquisition time but access to more redundant information [11]. For a system with $N_{E}=16$ electrodes, this yields 120 excitation patterns and 1920 measurements. The EIT system used for the dynamic experiments, as shown in Fig. 2(b), employs fast Fourier transform (FFT)-based frequency-division multiplexing instead of the time-consuming time-division multiplexing, and the superimposed signals of the 120 excitation patterns are discriminated by FFT, giving the boundary measurements simultaneously. This system applies constant voltage injection, the currents are obtained from voltages measured across resistors located in series in the voltage excitation circuit, and a detailed representation of the system can be found in [14] and [15]. The voltages are measured against the same ground as the voltage injection electrodes. The ground value is defined by the requirement that the sum of all voltages on the boundary is always zero.

The GRM allows determining the density of the investigated medium by measuring the attenuation of a radiation beam. The GRM installed at the USN rig (see Fig. 1) operates with a single beam projection, which gives access to an effective density along the beam path. Calibration has to be performed with measurements on both full and empty pipes to determine bounds on the measured density. In this study, the GRM provides a cross-sectional effective phase fraction $\beta$, which is used as a reference to assess the performance of the proposed EIT-based method. Given a measured GRM density $\rho_{m}$, the cross-sectional water phase fraction $\beta$ is calculated as $\beta=\left(\rho_{m}-\rho_{g}\right) /\left(\rho_{w}-\rho_{g}\right)$, with $\rho_{w}$ and $\rho_{g}$ being the density of water and air, respectively. 


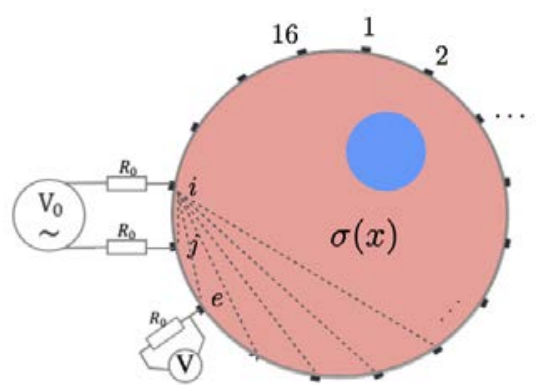

(a)

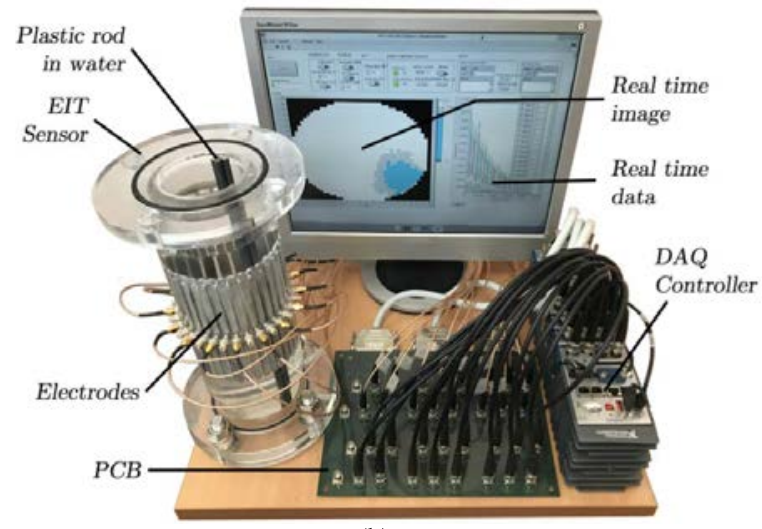

(b)

Fig. 2. EIT system employed on the USN multiphase flow rig. (a) Schematic of an EIT architecture using the full-scan excitation strategy for $N_{E}=16$ electrodes. Voltage excitation at electrode pair $(i, j)$ and voltage measurement $V_{e}$ across the constant resistor $R_{0}$ at electrode $e$. (b) High-speed EIT system developed at the LTHC and implementing the full-scan strategy, adapted from [15].

\section{B. Test Matrix}

The USN rig is operated in the horizontal configuration, and only water and air are injected during the experiments. The mass flow rate of water ranges from 1.58 to $79.43 \mathrm{~kg} / \mathrm{min}$ and of air from 0.1 to $5.0 \mathrm{~kg} / \mathrm{min}$. The electrical conductivity of air is negligible compared to that of water.

For horizontal air-water two-phase flows, the typical regimes include bubble, stratified smooth, stratified wavy, plug, slug, annular, intermittent, and mist flows (see [16] and [17]). Yet, in this study, only the stratified smooth, stratified wavy, plug, slug, and annular flows [see Fig. 3(a)] are considered because of the chosen input mass flow rates.

The flow regime map of Mandhane et al. [18] for a horizontal pipe with a $50-\mathrm{mm}$ diameter is used to select the experimental water and air mass flow rates. A total of 80 experimental sets are considered, consisting of approximately $2 / 5$ of intermittent flows (plug and slug), 2/5 of stratified flows, and $1 / 5$ of annular flow. The flow conditions are assessed by visual inspections during the experiments and reported on the flow regime map (test matrix) of Fig. 3(b).

Our EIT system can perform measurements at a frame rate up to 3906 frames/s [15], but, during each experiment, we selected a rate of 100 frames/s over $30 \mathrm{~s}$ as we found it is sufficient to capture the characters of the flow for the various phase injection conditions considered. A higher frame rate would not bring additional information while requiring more computational power. The GRM operates during $60 \mathrm{~s}$ at
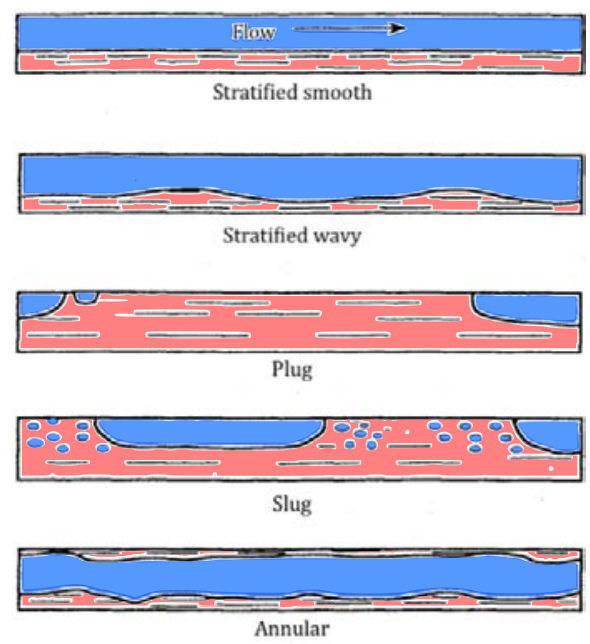

(a)

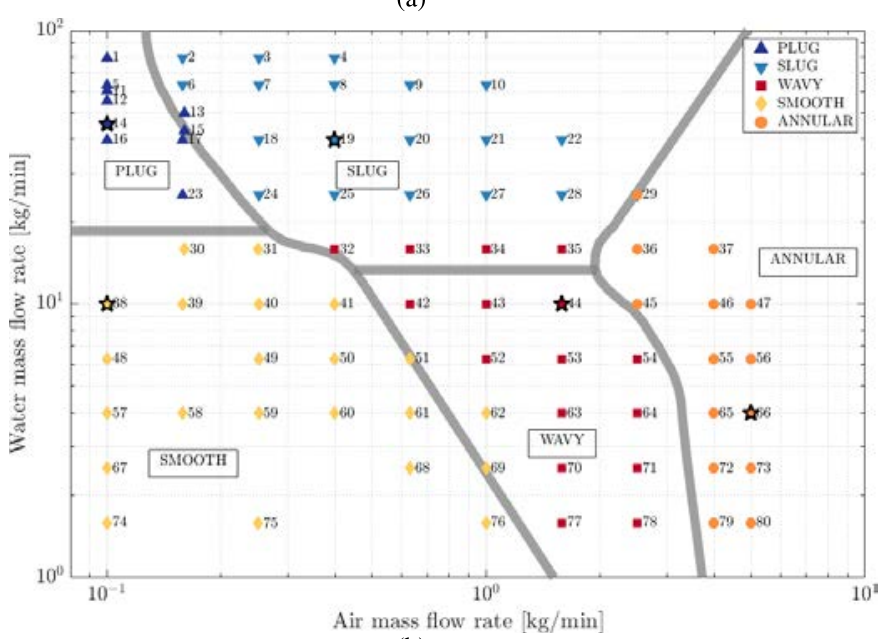

(b)

Fig. 3. Flow regimes investigated at the USN rig. (a) Horizontal two-phase flow regimes considered (blue indicates air). Image adapted from [16]. (b) Test matrix with the 80 experimental sets (colored symbols). The indicated flow regimes are assessed here from visual inspections. Stars indicate the experiments selected in Fig. 6. Thick gray lines indicate transition zones.

an acquisition rate of $20 \mathrm{~Hz}$, i.e., at a rate of five times slower than the EIT measurements.

\section{Methodology}

\section{A. Image Reconstruction Method}

The EIT inverse problem consists of determining the conductivity distribution $\sigma(x)$ that characterizes the medium considered. This is a nonlinear ill-posed inverse problem, which can be solved in a discrete setting using, e.g., a finite-element implementation and a cost functional minimization procedure. As is well-known, the resulting EIT-based images are usually oversmoothed using $L^{2}$-norm-based reconstruction methods. In addition, for the horizontal two-phase flows investigated here, the nonconductive air phase tends to gather on the top of the pipe, which distorts the electric field severely and, thus, impacts reconstructions.

In this study, the Newton's One-Step Error Reconstructor (NOSER) method [19] is used to form the EIT-based images. Although there are alternative and more advanced imaging techniques available, the NOSER method is adopted 
here as it is both simple and robust, and easily amenable to real-time reconstructions, making it suitable for the complex phase distribution patterns encountered in the dynamic experiments considered. In addition, the use of this simple method aims at highlighting the interest of the eigenvalue correlation method for improving flow visualizations. In NOSER, considering a linearized $L^{2}$-norm cost functional with the Tikhonov regularization, a discrete conductivity distribution $\sigma$ is computed through a single Gauss-Newton iteration as

$\boldsymbol{\sigma}=\boldsymbol{\sigma}_{\text {ref }}+\Delta \boldsymbol{\sigma}$ with $\Delta \boldsymbol{\sigma}=-\left(\boldsymbol{J}^{T} \boldsymbol{J}+\alpha \operatorname{diag}\left(\boldsymbol{J}^{T} \boldsymbol{J}\right)\right)^{-1} \boldsymbol{J}^{T} \Delta \boldsymbol{V}$

where $\sigma_{\text {ref }}$ is a reference conductivity field, $\boldsymbol{J}$ is the Jacobian matrix of the discrete forward operator, and $\Delta \boldsymbol{V}$ is the difference between the measurements and the boundary voltages associated with the reference conductivity $\sigma_{\text {ref }}$. Moreover, $\alpha \in$ $\mathbb{R}$ is a penalty coefficient chosen based on the so-called " $\mathrm{L}$ curve" method [20]. The matrix $\left(\boldsymbol{J}^{T} \boldsymbol{J}+\alpha \cdot \operatorname{diag}\left(\boldsymbol{J}^{T} \boldsymbol{J}\right)\right)^{-1} \boldsymbol{J}^{T}$ can be precalculated offline and then used for online real-time imaging.

In this study, $\sigma_{\text {ref }}$ is taken as the conductivity of water filling the pipeline, and the reconstructed conductivity $\sigma$ is computed on a spatial grid of $39 \times 39$ points. The cross-sectional EIT-based images are displayed and updated every $0.1 \mathrm{~s}$, as permitted by the computational capabilities of our acquisition system.

\section{B. Normalized Impedance Matrix}

Fig. 2(a) shows a schematic of the EIT system, with voltage excitation at an electrode pair $(i, j)$ and voltage measurement $V_{e}$ across a constant resistor $R_{0}$ at electrode $e$, and the current measurement $I_{e}$ being $I_{e}=V_{e} / R_{0}$. The equivalent circuit model of the system is depicted in Fig. 4: the combination of the charge transfer resistance $R_{e}$ and the double layer capacitance $C_{e}$ contributes to the contact impedance, $Z_{i, j}$ being the bulk impedance, connected in a cascade way with $R_{0}$. In this study, only the resistive part of the medium bulk impedance is considered. Darnajou et al. [14] showed that the contact impedance effects can be neglected since a continuous excitation signal is used. Considering that the current measurements at all of the $N_{E}$ electrodes sum up to zero, the current $I_{i, j}$ flowing through the closed circuit is $I_{i, j}=-\sum_{e=1}^{N_{E}} V_{e} / R_{0}$, with the exciting electrodes being excluded in the summation over the index $e$. Finally, the bulk impedance is calculated directly as $Z_{i, j}=V_{0} / I_{i, j}-2 R_{0}$, where $V_{0}$ is the excitation voltage.

For each pair of excitation electrodes $(i, j)$, there is a corresponding bulk impedance $Z_{i, j}$, which constitutes an entry of a symmetric impedance matrix $\boldsymbol{Z}=\left(Z_{i, j}\right)_{1 \leq i, j \leq N_{E}} \in \mathbb{R}^{N_{E} \times N_{E}}$. However, to reduce the dependencies on the geometric parameters of the sensor and on the background conductivity, a version $\hat{Z}_{i, j}=\left(Z_{i, j}-Z_{i, j}^{\text {ref }}\right) / Z_{i, j}^{\text {ref }}$ normalized relatively to the bulk impedance $Z_{i, j}^{\text {ref }}$ of the reference conductivity $\sigma_{\text {ref }}$ is preferred to $Z_{i, j}$. This leads to a normalized impedance matrix (NIM) of the form: $\hat{Z}=\left(\hat{Z}_{i, j}\right)_{1 \leq i, j \leq N_{E}}$.

Using numerical and experimental benchmarking, Dang et al. [13] evidenced that the phase fraction can

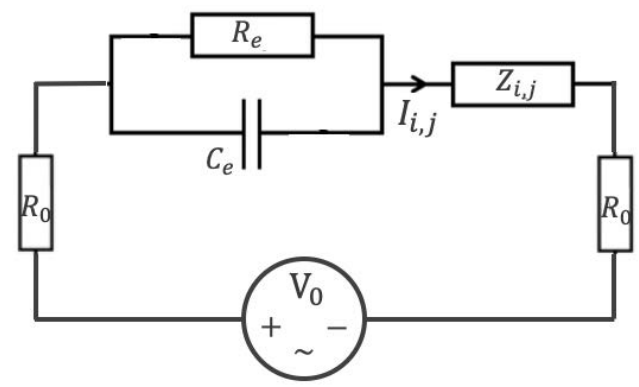

Fig. 4. Equivalent closed circuit of the EIT system, with $V_{0}$ being the excitation voltage, $R_{e}$ and $C_{e}$ contributing to the contact impedance, and $Z_{i, j}$ being the measured medium bulk impedance.

be estimated from the eigenvalues of $\hat{\mathbf{Z}}$. In particular, the maximum eigenvalue $\lambda^{\max }$, referred to as the so-called leading eigenvalue, exhibits strong correlations with the void fraction, provided that the current state of the flow regime is known beforehand. In this context, our objective is to use such correlations in a procedure that aims at improving EIT images through the determination of the interface between phases based on the void fraction estimated from the principal component analysis of the NIM.

\section{IMPROVING EIT-BASED FLOW VISUALIZATIONS}

In this section, we expose our approach combining EIT-based image reconstructions and the eigenvalue correlation method. The proposed flow visualization procedure is as follows.

1) For the configuration considered and the EIT system employed, the correlation between the NIM leading eigenvalue and the AVF is investigated for different flow regimes, from which a calibration diagram is constituted.

2) For a given experiment, the temporal evolution of the NIM leading eigenvalue $\lambda^{\max }$ is analyzed and associated with a raw image guess, from which the flow regime is identified.

3) Cross-sectional EIT-based raw image reconstructions are stacked axially to form a time-dependent tomogram.

4) Knowing the flow regime from $B$, the calibration diagram of $\mathrm{A}$ is used to estimate the AVF, thus leading to a threshold value that is used to binarize the EIT tomogram of $\mathrm{C}$.

Remark 1: In this study, the raw image reconstructions are computed using the NOSER method (see Section III-A). It should be noted that the flow visualization improvement procedure above does not rely on that method specifically, and alternative image reconstruction algorithms can be used as well.

Remark 2: The threshold value determined in step D is here applied to all the images along the time sequence, i.e., the AVF is estimated only once for a given experimental sequence. Yet, the proposed approach can be applied to each cross-sectional image, i.e., at each time step, if necessary, with the knowledge of the current state of the flow regime.

Each of the steps of the procedure above is now detailed and illustrated on the experimental data set obtained on the USN loop. 


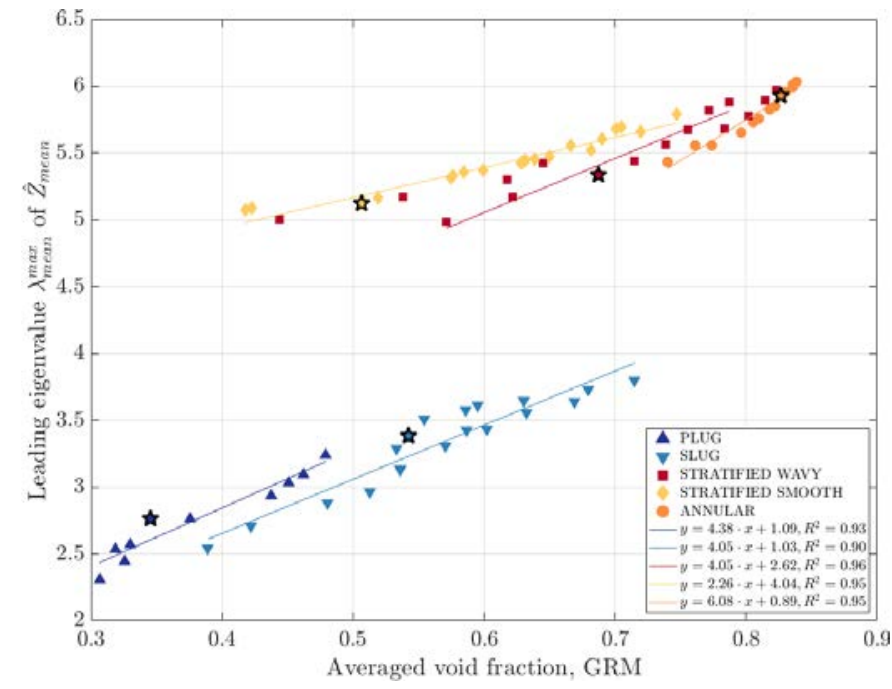

Fig. 5. System calibration diagram: eigenvalue $\lambda_{\text {mean }}^{\max }$ versus reference AVF from GRM, with indication of the flow regimes. Stars indicate the experiments selected in Fig. 6.

\section{A. System Calibration From NIM Leading Eigenvalue and AVF Correlation}

The Maxwell-Hewitt relation can be used to approximate the phase fraction from EIT data (see [8]). However, this relation is only applicable when the background medium is continuous and the nonconductive phase is monodispersed, which is not the case for the horizontal two-phase flows considered here. To the best of our knowledge, there are only a few methods reported on void fraction estimation in such configurations based on EIT data only. Note, however, that a dual-modality ECT/EIT measurement-based approach has been used in [21] for image segmentation. Here, the correlation between the NIM leading eigenvalue and the AVF is investigated from EIT raw data, with the reference AVF being calculated by averaging the GRM void fraction for each experimental set. This leads to a system calibration diagram applicable to subsequent (i.e., unknown) flow conditions.

Given a flow regime calibration condition, a number $N_{F}$ of EIT measurement frames are acquired, each being associated with a normalized impedance matrix $\hat{\boldsymbol{Z}}_{f}$. A mean NIM $\hat{\boldsymbol{Z}}_{\text {mean }}$ is then computed as

$$
\hat{\boldsymbol{Z}}_{\text {mean }}=\frac{1}{N_{F}} \sum_{f=1}^{N_{F}} \hat{\boldsymbol{Z}}_{f}
$$

whose leading eigenvalue is denoted as $\lambda_{\text {mean }}^{\max }$. The matrix $\hat{Z}_{\text {mean }}$ then contains information on the measured flow, which is extracted by a principal component analysis (see [13]).

This is applied to the system investigated at USN, and Fig. 5 represents the calibration diagram thus obtained. The eigenvalue $\lambda_{\text {mean }}^{\max }$ is plotted against the reference GRM void fraction for 80 of calibration experiments represented in Fig. 3(b), along with the determined flow regimes.

Remark 3: Here, each calibration experiment is monitored with the same number $N_{F}=3000$ of EIT frames. Yet, different numbers of frames can be considered in practice from one experiment to another.
For each flow regime, a linear correlation is found between $\lambda_{\text {mean }}^{\max }$ and the AVF with a high confidence, which agrees with the conclusions of Dang et al. [13], which were based on numerical and experimental benchmarking. It should be noted that estimating this correlation for stratified wavy flows is more involved as the transitions between smooth, wavy, and annular flows are continuous, which makes them difficult to discriminate from one another, even through visual inspection. Therefore, the experiments associated with such transition zones [see Fig. 3(b)] are excluded from the assessment of the leading eigenvalue correlation.

For a given EIT system configuration, due to the normalization of the impedance matrix, the correlation between the eigenvalue $\lambda_{\text {mean }}^{\max }$ and the $\mathrm{AVF}$ is a robust characteristic clearly related to the property of the conductive (liquid) phase. Finally, while we only make use of the behavior of the leading eigenvalue as a simple and robust criterion in this study, it has been underlined in [13] that alternative NIM-based metrics can be employed to investigate the phase fraction.

\section{B. Temporal Evolution of the Leading Eigenvalue}

With the calibration diagram at hand, we now investigate a given experiment. In this second step, the leading eigenvalue $\lambda^{\max }$ of the normalized impedance matrix $\hat{\boldsymbol{Z}}_{f}$ is computed for each frame $f=1, \ldots, N_{F}$ of the experiment considered. Fig. 6 then shows the temporal evolution of $\lambda^{\max }$ along time (or, equivalently, frame $f$ ) for the five selected experiments identified in Figs. 3(b) and 5, which corresponds to different flow regimes. The eigenvalue $\lambda^{\max }$ for the stratified and annular flows is plotted together to emphasize the differences in their behaviors. In Fig. 6(a) and (b), the intermittent flows present characteristic features: $\lambda^{\max }$ varies significantly between 0 and 4, while the slug and plug flows are distinguished from one another by the value of $\lambda^{\max }$ in between the plateaus. Moreover, the plug flow is associated with more abrupt variations of $\lambda^{\max }$. This can be related to the different behaviors of the plug and slug flows, with the small plugs encountered between long plugs compared to the intensive bubbles entrained in the water bulk between the slugs [see Fig. 3(a)]. As to the stratified and annular flows, Fig. 6(c) shows large and chaotic variations of $\lambda^{\max }$ for wavy flow and, similarly, for the annular flow but at higher values, the smooth flow being associated with a steady level.

In addition, Fig. 7 shows the plots of the raw cross-sectional image reconstructions $\Delta \sigma=\left(\sigma-\sigma_{\text {ref }}\right)$ obtained with the NOSER method (or "image guesses") for the experiments considered, and computed at the time $t=5.3 \mathrm{~s}$ (indicated by black dashed line in Fig. 6). We recall that $\sigma_{\text {ref }}$ is chosen as the conductivity of water. The range of $\Delta \sigma$ is renormalized to [ $\left.\begin{array}{ll}-1 & 1\end{array}\right]$ to show clearly the interface leading to a different colormap. Comparison of Fig. 7(a) and (b) illustrates how a high value of $\lambda^{\max }$ correlates with a high void fraction. Note that, for the stratified and annular flows, the voltage measurements over the top electrodes are noisy, as these are only partially covered by the conductive water, thus leading to inaccurate reconstructions in this region 


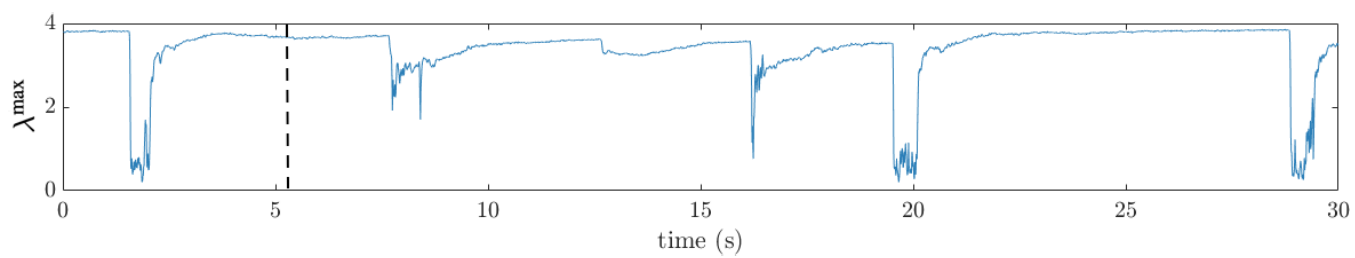

(a)

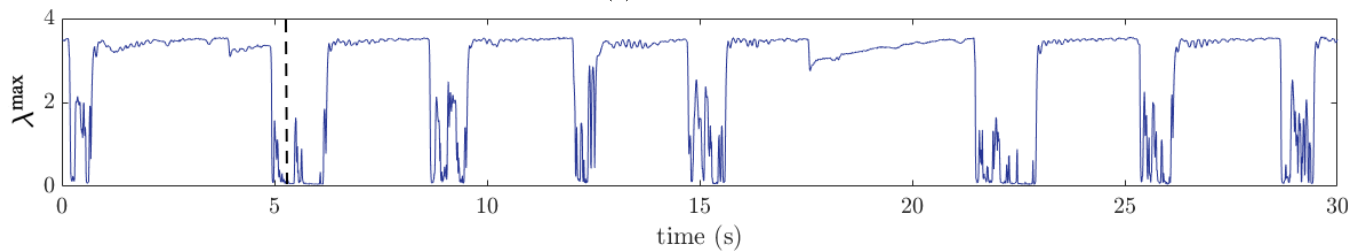

(b)

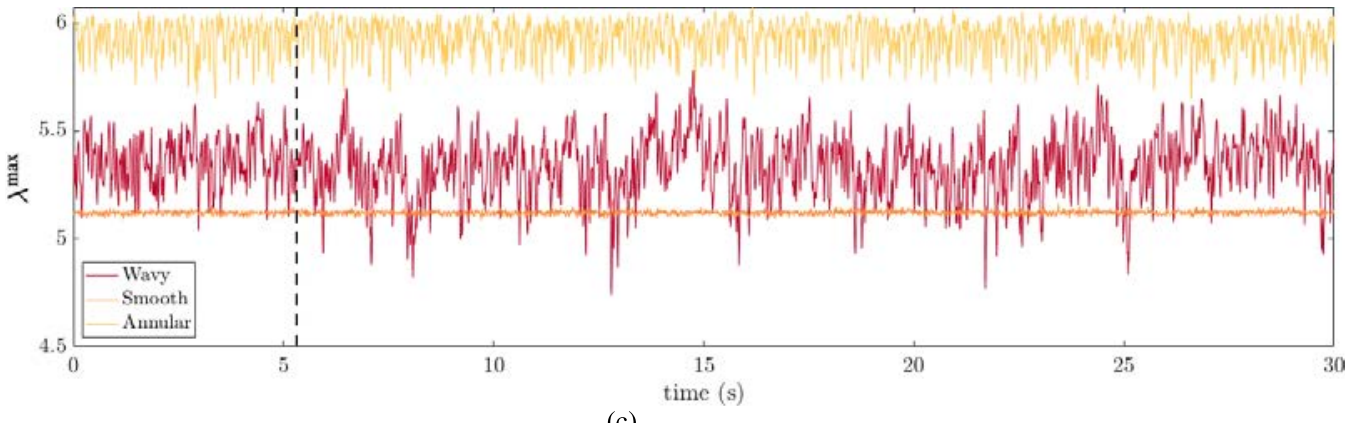

(c)

Fig. 6. Temporal evolution of the leading eigenvalue $\lambda^{\max }$ of $\hat{\boldsymbol{Z}}_{f}$ along time (or frame $f$ ) for the five selected experiments identified in Figs. 3(b) and 5. The cross-sectional images at time $t=5.3 \mathrm{~s}$ (vertical dashed line) are shown in Fig. 7. (a) Slug flow. (b) Plug flow. (c) Stratified and annular flows.

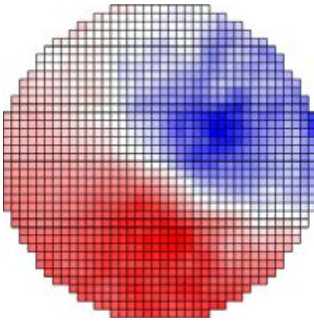

(a)

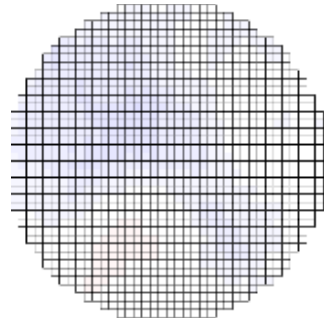

(b)

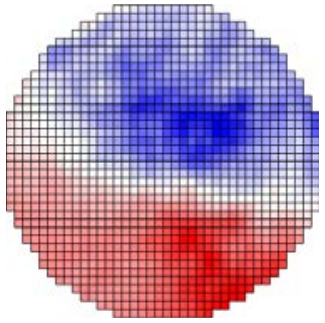

(c)

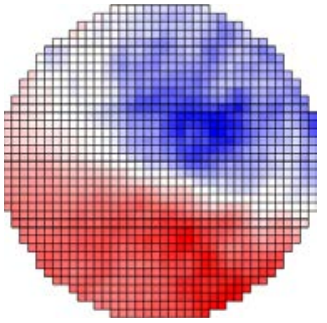

(d)

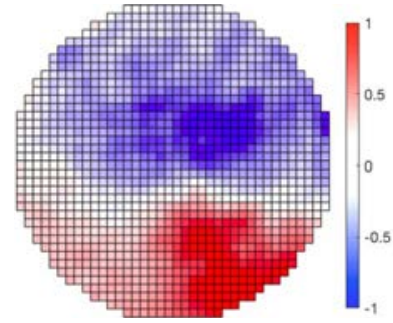

(e)

Fig. 7. Renormalized cross-sectional image reconstructions $\Delta \sigma=\left(\sigma-\sigma_{\text {ref }}\right)$ for the selected cases and at the time $t=5.3 \mathrm{~s}$, indicated by the black dashed lines in Fig. 6. (a) Slug. (b) Plug. (c) Stratified wavy. (d) Stratified smooth. (e) Annular.

[see Fig. 7(e)]. Finally, the time being fixed, the reconstructions of Fig. 7(c)-(e) are very similar, while the associated flows can be clearly discriminated from the temporal evolution of $\lambda^{\text {max }}$, as discussed previously.

These results highlight that it is the combination of both the temporal evolution of $\lambda^{\max }$, as shown in Fig. 6, and raw image guesses-based observations, as shown in Fig. 7, which allows determining the flow pattern with certainty for a given experiment.

\section{Stacked EIT Tomogram}

Considering a time sequence of EIT measurements, the instantaneous reconstructed cross-sectional images are fragmented and not representative of the whole flow, as seen previously in Fig. 7. Instead, the axially stacked tomogram is more suitable to flow visualization. Therefore, the pixels on the central vertical line of the cross-sectional images are extracted and stacked along time (or frame $f$ ), forming a tomogram for the experiment considered.

Here, the comparison is made with the reference GRM information and its measurement $\beta$ of the water phase fraction (see Section II-A). As, for horizontal flows, it is reasonable to assume that, in most cases, the air gathers at the top of the pipe, except that the front of the slug contains intensive bubbles entrained in the water bulk, then the water phase forms a segment at the bottom with area $A$. With this assumption, we get $\beta=A / \pi r_{0}^{2}$, where $r_{0}$ is the radius of the pipeline, from which the time-dependent height $h$ of the water phase is obtained. This results in a reference GRM tomogram for each experimental set.

Both the raw EIT tomogram and the reference GRM one are shown in Fig. 8 (top and bottom, respectively) for three distinctive flow regimes (slug, stratified wavy, and annular) among these considered previously. The time resolutions of the EIT and GRM tomograms are 10 and $50 \mathrm{~ms}$, respectively. 

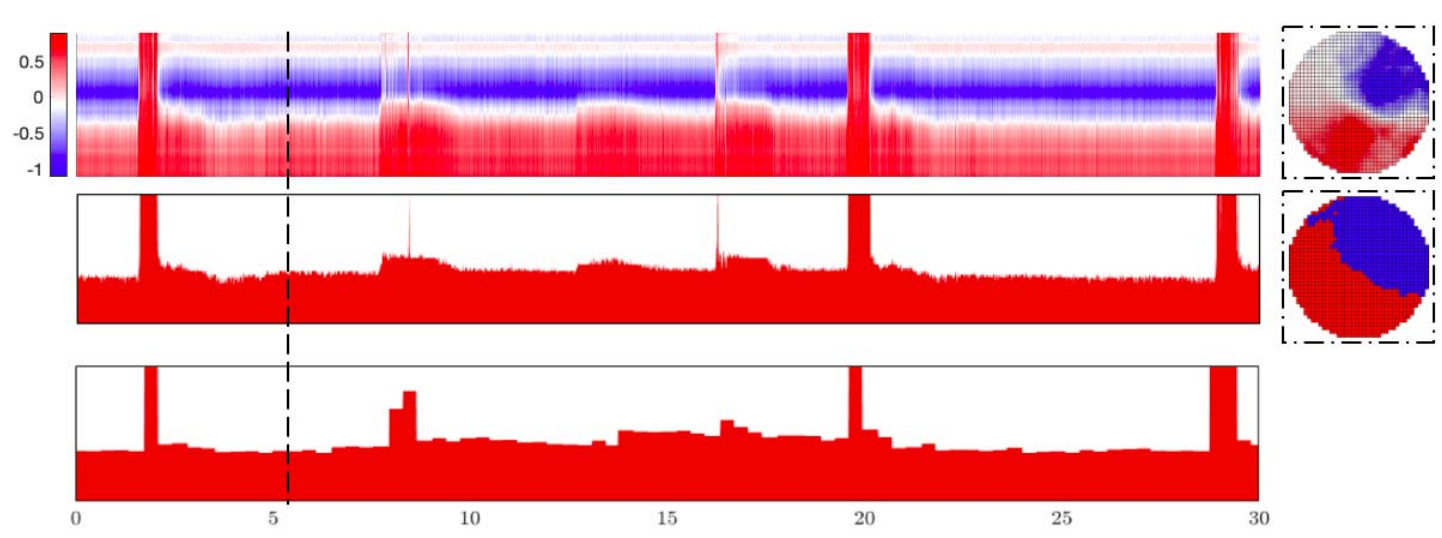

(a)
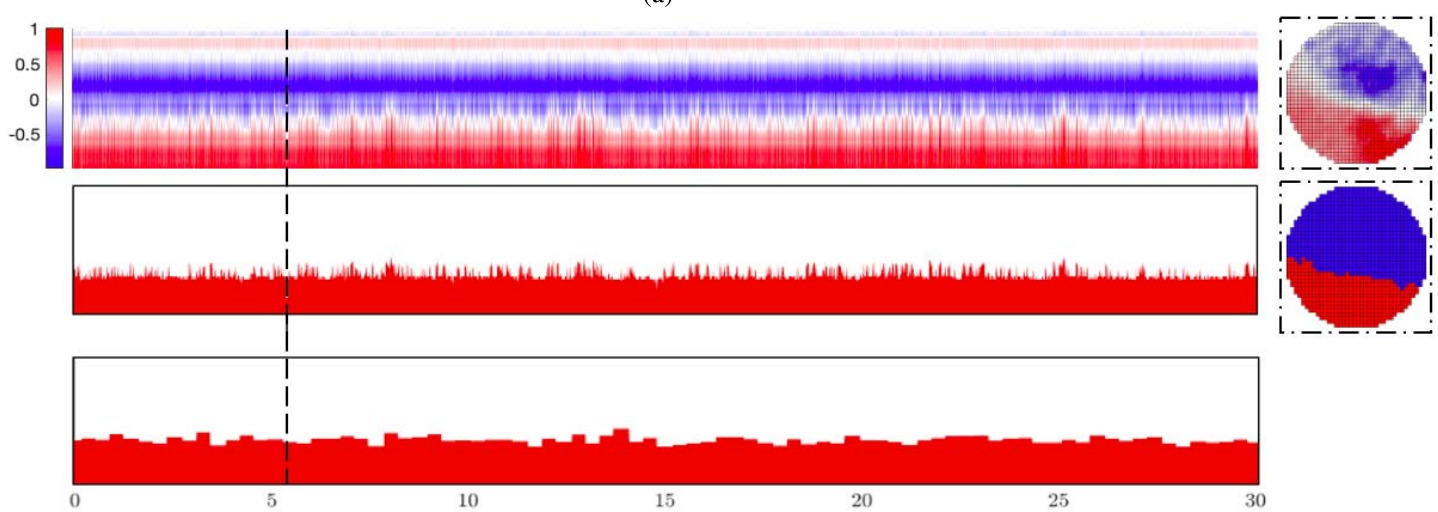

(b)
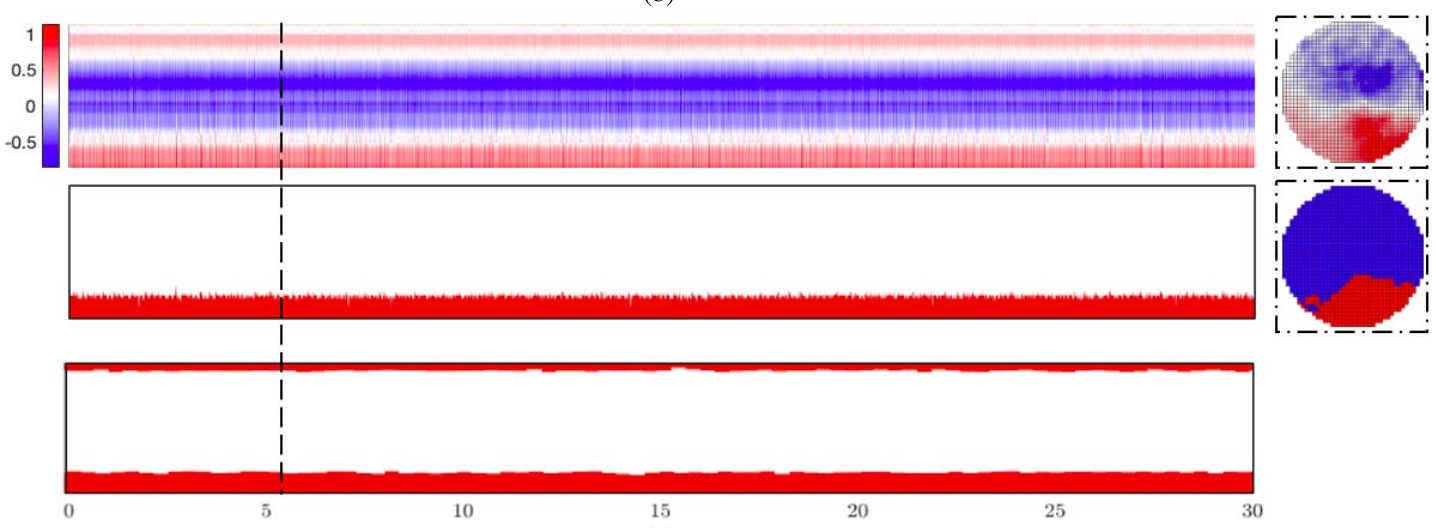

(c)

Fig. 8. Flow distributions along the longitudinal section: (top) raw EIT distributions, i.e., renormalized $\Delta \sigma$, (middle) binarized EIT distributions, and (bottom) reference GRM distributions, for the experimental sets considered of slug, stratified wavy, and annular flows. The abscissae represents the measurement time in second (or frame $f$ ). The vertical dashed lines corresponds to $t=5.3 \mathrm{~s}$ with the corresponding cross-sectional images shown in the right. (a) Slug flow. (b) Stratified wavy flow. (c) Annular flow.

In Section IV-D, we finally show how these EIT tomograms can be binarized using the AVF estimated by the eigenvalue correlation method.

\section{Tomogram Binarization and Flow Visualization}

Classically, in EIT, the reconstructed images do not give a precise indication of the phase distribution because of their oversmoothness. Yet, a threshold value $\sigma_{\text {th }}$ can be computed using the eigenvalue correlation method: the mean leading eigenvalue $\lambda_{\text {mean }}^{\max }$ is computed for the experiment considered, and having determined the flow regime at step B, the calibration diagram of step A (see Fig. 5) is then used to estimate the AVF. The threshold $\sigma_{\text {th }}$ is then defined so that the phase fraction in the binarized EIT distribution matches the estimated phase distribution (see Remark 2). This procedure is exemplified in the experiment considered with the binarized EIT distributions shown in Fig. 8 (middle).

Overall, there is an excellent agreement between the air-water interface in the binarized EIT distributions and in the reference GRM ones. In Fig. 8(a), the characteristic intermittent behavior of the slug flow can be clearly identified from both the EIT and GRM distributions. Note also the strong correlation between the temporal evolution of $\lambda^{\text {max }}$ in Fig. 6(a) and the phase distribution variations in Fig. 8(a). Similarly, the fluctuations at the air-water interface in the stratified wavy flow are well captured in the binarized EIT distribution of 
Fig. 8(b). For the annular flow, the assumption that the air phase gathers at the top of the pipe does not hold anymore. Paras and Karabelas [22] made some observation-based predictions of the film circumferential thickness distribution for a horizontal pipe. The predicted ratio of the top and bottom film thicknesses is used here to form the reference GRM distribution of Fig. 8(c). However, the thin top water film is not well reconstructed using EIT.

To achieve a quantitative comparison, the time evolution of the phase fraction is computed from the binarized EIT distribution and compared to the GRM measurement. The mean relative errors for the three cases considered are $4.75 \%$, $4.23 \%$, and $3.67 \%$, respectively. Generally speaking, to explain the small observed discrepancies between the EIT and GRM distributions, certain phenomena should be taken into account. First, EIT measurements have a 3-D nature that tends to induce a local (volume) averaging in images. Second, the temporal resolution of EIT is five times higher than that of GRM, which may introduce extra error. Finally, the noisy voltage measurements at the top electrodes induce high conductivity artifacts in the vicinity of the latter in EIT images and tomograms, as previously discussed. We have observed that discarding the top measurements tends to reduce this effect.

\section{CONClusion}

This article provides a proof of concept of the eigenvalue correlation method described in [13] and demonstrates its applicability in classifying and detecting flow regimes using the pilot scale with data from multiphase flow rig under different flow conditions. The data acquired during extensive experiments involving various combinations of air and water with varying fractions and velocities were used to identify the flow regime and estimating the volume AVF using sensor data fusion. Redundant EIT measurements are acquired using the full-scan excitation strategy, which enables to directly estimate the void fraction without performing image reconstruction. Yet, the extracted flow features are further integrated into EIT tomograms to improve flow visualizations.

The proposed eigenvalue correlation method yields excellent results, with the correlation between the leading eigenvalue of the normalized impedance matrix and the void fraction being consistent given the EIT system configuration. It can be precalibrated as a chart then usable for void fraction estimation by direct lookup. The estimation error is low, with linear correlation fits for the different flow regimes. Moreover, the temporal evolution of the leading eigenvalue coupled with a raw image guess gives a clear indication of the flow regime, which can be used as prior information for the eigenvalue correlation method to estimate the volume AVF. The estimated AVF can then be applied to adjust the phase interface in the EIT tomogram, leading to a more reliable indication of the phase distribution.

For future studies, applying the proposed method to vertical air-water two-phase flows would be interesting, as most of the peripheral electrodes will be in contact with the conductive phase, thus preventing the issues related to the uncovered electrode. Furthermore, considering the substantially large amount of data generated by EIT, i.e., the NIM for each frame, it is appealing to apply a statistical learning approach to estimate the void fraction using the proposed method.

\section{ACKNOWLEDGMENT}

Multiphase experiments involving multimodal sensors, including the electrical impedance tomography (EIT) and gamma-ray meter (GRM), were performed in the process lab at the University of South-Eastern Norway (USN) with the help of Senior Engineer, Fredrik Hansen, and Master Student, Aleksander Tokle Poevrud.

\section{REFERENCES}

[1] G. Ricciardi, M. J. Pettigrew, and N. W. Mureithi, "Fluidelastic instability in a normal triangular tube bundle subjected to air-water cross-flow," J. Pressure Vessel Technol., vol. 133, no. 6, Dec. 2011, Art. no. 061301.

[2] T. J. Heindel, "A review of X-ray flow visualization with applications to multiphase flows," J. Fluids Eng., vol. 133, no. 7, Jul. 2011, Art. no. 074001.

[3] H. F. Velasco Peña and O. M. H. Rodriguez, "Applications of wire-mesh sensors in multiphase flows," Flow Meas. Instrum., vol. 45, pp. 255-273, Oct. 2015.

[4] M. V. Sardeshpande, S. Harinarayan, and V. V. Ranade, "Void fraction measurement using electrical capacitance tomography and high speed photography," Chem. Eng. Res. Des., vol. 94, pp. 1-11, Feb. 2015.

[5] K. Perera, W. Kumara, F. Hansen, S. Mylvaganam, and R. W. Time, "Comparison of gamma densitometry and electrical capacitance measurements applied to hold-up prediction of oil-water flow patterns in horizontal and slightly inclined pipes," Meas. Sci. Technol., vol. 29, no. 6, 2018, Art. no. 065102 .

[6] B. H. Brown, "Medical impedance tomography and process impedance tomography: A brief review," Meas. Sci. Technol., vol. 12, no. 8, pp. 991-996, Aug. 2001.

[7] M. Sharifi and B. Young, "Electrical resistance tomography (ERT) applications to chemical engineering," Chem. Eng. Res. Des., vol. 91, no. 9, pp. 1625-1645, Sep. 2013.

[8] D. L. George, J. R. Torczynski, K. A. Shollenberger, T. J. O’Hern, and S. L. Ceccio, "Validation of electrical-impedance tomography for measurements of material distribution in two-phase flows," Int. J. Multiphase Flow, vol. 26, no. 4, pp. 549-581, Apr. 2000.

[9] J. Jia, H. Wang, and D. Millington, "Electrical resistance tomography sensor for highly conductive oil-water two-phase flow measurement," IEEE Sensors J., vol. 17, no. 24, pp. 8224-8233, Dec. 2017.

[10] A. Dupre, "Electrical impedance tomography for void fraction measurements of harsh two-phase flows: Prototype development and reconstruction techniques," Ph.D. dissertation, Dept. Physique et Sci. de la Matière, École Centrale de Marseille, Marseille, France, 2017.

[11] C. Dang, "Imaging and fast features extraction of two-phase flows using electrical impedance tomography," Ph.D. dissertation, École Centrale de Marseille, Marseille, France, 2020.

[12] W. Fang and E. Cumberbatch, "Matrix properties of data from electrical capacitance tomography," J. Eng. Math., vol. 51, no. 2, pp. 127-146, Feb. 2005.

[13] C. Dang, M. Darnajou, C. Bellis, G. Ricciardi, H. Schmidt, and S. Bourennane, "Numerical and experimental analysis of the correlation between EIT data eigenvalues and two-phase flow phase fraction," Meas. Sci. Technol., vol. 31, no. 1, Jan. 2020, Art. no. 015302.

[14] M. Darnajou, A. Dupré, C. Dang, G. Ricciardi, S. Bourennane, and C. Bellis, "On the implementation of simultaneous multi-frequency excitations and measurements for electrical impedance tomography," Sensors, vol. 19, no. 17, p. 3679, Aug. 2019.

[15] M. Darnajou et al., "High speed EIT with multifrequency excitation using FPGA and response analysis using FDM," IEEE Sensors J., vol. 20, no. 15, pp. 8698-8710, Aug. 2020.

[16] Y. Chen, W. P. Jepson, and H. J. Chen, "Effects of multiphase flow on corrosion inhibitor," in Proc. CORROSION, 1999, Paper 12.

[17] K. Perera, C. Pradeep, S. Mylvaganam, and R. W. Time, "Imaging of oilwater flow patterns by electrical capacitance tomography," Flow Meas. Instrum., vol. 56, pp. 23-34, Aug. 2017.

[18] J. Mandhane, G. Gregory, and K. Aziz, "A flow pattern map for gasliquid flow in horizontal pipes," Int. J. Multiphase Flow, vol. 1, no. 4, pp. 537-553, 1974. 
[19] M. Cheney, D. Isaacson, J. C. Newell, S. Simske, and J. Goble, "NOSER: An algorithm for solving the inverse conductivity problem," Int. J. Imag. Syst. Technol., vol. 2, no. 2, pp. 66-75, 1990.

[20] M. Vauhkonen, "Electrical impedance tomography and prior information,” Ph.D. dissertation, Dept. Appl. Phys., Kuopio Univ., Kuopio, Finland, 1997.

[21] Q. Wang, M. Wang, K. Wei, and C. Qiu, "Visualization of gas-oil-water flow in horizontal pipeline using dual-modality electrical tomographic systems," IEEE Sensors J., vol. 17, no. 24, pp. 8146-8156, Dec. 2017.

[22] S. V. Paras and A. J. Karabelas, "Properties of the liquid layer in horizontal annular flow," Int. J. Multiphase Flow, vol. 17, no. 4, pp. 439-454, Jul. 1991. 